\title{
Deriving generic telescope use cases for the Cherenkov Telescope Array
}

\author{
I. Oya, M. Füßling, J. A. Hinton, A. Mitchell, G. Tosti, et \\ al.
}

I. Oya, M. Füßling, J. A. Hinton, A. Mitchell, G. Tosti, F. Dazzi, "Deriving generic telescope use cases for the Cherenkov Telescope Array," Proc. SPIE 10705, Modeling, Systems Engineering, and Project Management for Astronomy VIII, 107050M (10 July 2018); doi: 10.1117/12.2312681

SPIE Event: SPIE Astronomical Telescopes + Instrumentation, 2018, Austin, Texas, United States 


\title{
Deriving generic telescope use cases for the Cherenkov Telescope Array
}

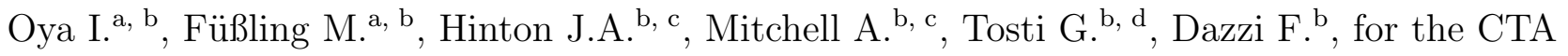 \\ Consortium $^{\mathrm{e}}$, and the CTA Observatory \\ ${ }^{a}$ DESY, Zeuthen, Germany \\ ${ }^{\mathrm{b}} \mathrm{CTAO}$ gGmbH, Heidelberg, Germany \\ ${ }^{\mathrm{c}}$ MPI für Kernphysik, Heidelberg, Germany \\ ${ }^{\mathrm{d}}$ University of Perugia, Italy \\ ehttp://www.cta-observatory.org
}

\begin{abstract}
The Cherenkov Telescope Array (CTA) is the next-generation atmospheric Cherenkov gamma-ray observatory. CTA will be deployed as two installations, one in the northern and the other in the southern hemisphere, containing dozens of telescopes of different sizes and designs, used for covering different energy domains. These telescopes interact with other systems (e.g. central observation execution software, infrastructure, etc.) fundamental for the Observatory operations. We have created a set of about 70 use cases (UCs) that describe the different type of interactions of a generic CTA telescope with its surrounding systems. These UCs describe different scenarios, from normal night operations to reactions to hazardous situations. Thanks to these UCs we can refine requirements, identify interfaces and specify the expected behaviour of the telescopes. The UCs are also an important ingredient to prepare the test cases for the integration and validation process of the telescopes into the CTA Observatory. This contribution summarises the methodology and tooling we have followed to identify and specify these UCs, as well as the main obtained results.
\end{abstract}

Keywords: Telescope, use case, software design, Cherenkov telescope

\section{INTRODUCTION}

The Cherenkov Telescope Array (CTA) is an initiative to build the next-generation atmospheric Cherenkov gamma-ray observatory. ${ }^{1}$ The CTA Observatory (CTAO) will consist of two facilities, one in the southern (close to the Paranal Observatory, Chile) and the other in the northern hemisphere (at the Roque de Los Muchachos Observatory, La Palma, Spain). The two sites will contain dozens of telescopes of different sizes, constituting one of the largest astronomical installations under development, and a factor twenty times larger than any ground based gamma-ray installation built so far. ${ }^{2-4}$

CTA will utilize telescopes of three sizes, which are required to optimize the instrument response in the CTA energy range (from a few tens of $\mathrm{GeV}$ to a few hundreds of $\mathrm{TeV}$ ). Different medium and small size telescope structure and camera designs have been prototyped, which contain some intrinsic differences such as the usage of single or dual-mirror reflectors, or different photo-detector technologies.

The CTA system will be decomposed in several top-level systems. ${ }^{5}$ The observations of a CTA installation are coordinated by the CTA Observation Execution System (OES). ${ }^{6}$ The OES will coordinate all operations involving multiple telescopes and/or telescope operation from a remote location*, storing all the acquired scientific and housekeeping data. Global observatory safety will be overseen by the Safety and Alarms System (SAS). Acquired data will be transferred, processed and archived by the Data Processing and Preservation Systems (DPPS), which will be hosted by large data centres in Europe.

Further author information:

I.O.: E-mail: igor.oya.vallejo@desy.de, Telephone: +49 3376277226

* remote: without a person physically present at the telescope location.

Modeling, Systems Engineering, and Project Management for Astronomy VIII

edited by George Z. Angeli, Philippe Dierickx, Proc. of SPIE Vol. 10705

107050M · @ 2018 SPIE · CCC code: 0277-786X/18/\$18

Proc. of SPIE Vol. 10705 107050M-1 
In order to prevent unnecessary system complexity and reduce future maintenance costs in the CTAO, a homogeneous interface to the different telescope types is necessary. The CTAO architecture team has described the generic behaviour of any CTAO telescope by means of use cases (UCs). These UCs are used to refine the existing set of functional requirements and provide invaluable input for defining the common software interface from OES and SAS to the telescopes.

This contribution describes the status and prospects in the creation of the CTAO generic telescope use cases. After introducing - from a very simplified perspective - what a CTA Cherenkov telescope is in Section 2, we present the methodology we have followed to create these UCs and the main results in Sections 3 and 4, respectively. Section 5 describes the lessons we have learned during this activity, and we close with the next steps planned around this task in Section 6.

\section{ANATOMY OF A CHERENKOV TELESCOPE}

An imaging atmospheric Cherenkov telescope (shortened to Cherenkov telescope hereafter) is an instrument designed to detect gamma-rays of cosmic origin. ${ }^{7}$ This telescope takes advantage of the fact that when gammarays enter Earth's atmosphere, they initiate cascades — or showers - of electrons, positrons and secondary gamma-rays. The charged particles created in these cascades produce fast flashes of visible light (of a few ns duration each) due to the Cherenkov effect. Other types of energetic particles such as protons and charged nuclei also create atmospheric showers, but in this case the shower has additional component beyond the electromagnetic part.

A Cherenkov telescope is an instrument that contains a Structure that can point to any position in the sky and that holds a reflector that focuses the light from Cherenkov showers into a photo-detector Camera. The analysis of these shower images permits the discrimination of showers originated from gamma-rays with those from other energetic particles, ${ }^{8}$ and it can be used to derive the direction and the energy of the gamma-ray that caused the electromagnetic shower. ${ }^{7}$ The logical decomposition of a generic CTA telescope is the following (See also Fig. 1):

- Telescope Structure: Light collector (mount and optics). Abbreviated to Structure hereafter. It is composed of software and hardware.

- Cherenkov Camera: Light detector. Abbreviated to Camera hereafter. It is composed of software and hardware.

- Telescope Manager: A software element that is responsible for making a telescope work as a coherent whole.

\section{METHODOLOGY}

We have created our generic telescope use cases following a methodology based on the prescriptions of A. Cockburn, ${ }^{9}$ using the Sparx Enterprise Architect $^{\dagger}$ (EA) modelling software. Our approach to identify and furnish the use cases is iterative and consists in the following steps:

1. Brainstorm a list of use case titles.

2. Organize the list of use cases into categories and display them in the form of Unified Modelling Language (UML) use case diagrams, which indicates the relationships between use cases, actors and CTAO systems. Figure 2 shows, as an example, one of these use case diagrams.

3. Produce a short description of what the use case does in natural language (one or a few sentences), identifying the scope of the use case. This description should reflect the main goal and interactions of the use case.

\footnotetext{
${ }^{\dagger}$ https://www.sparxsystems.com
} 


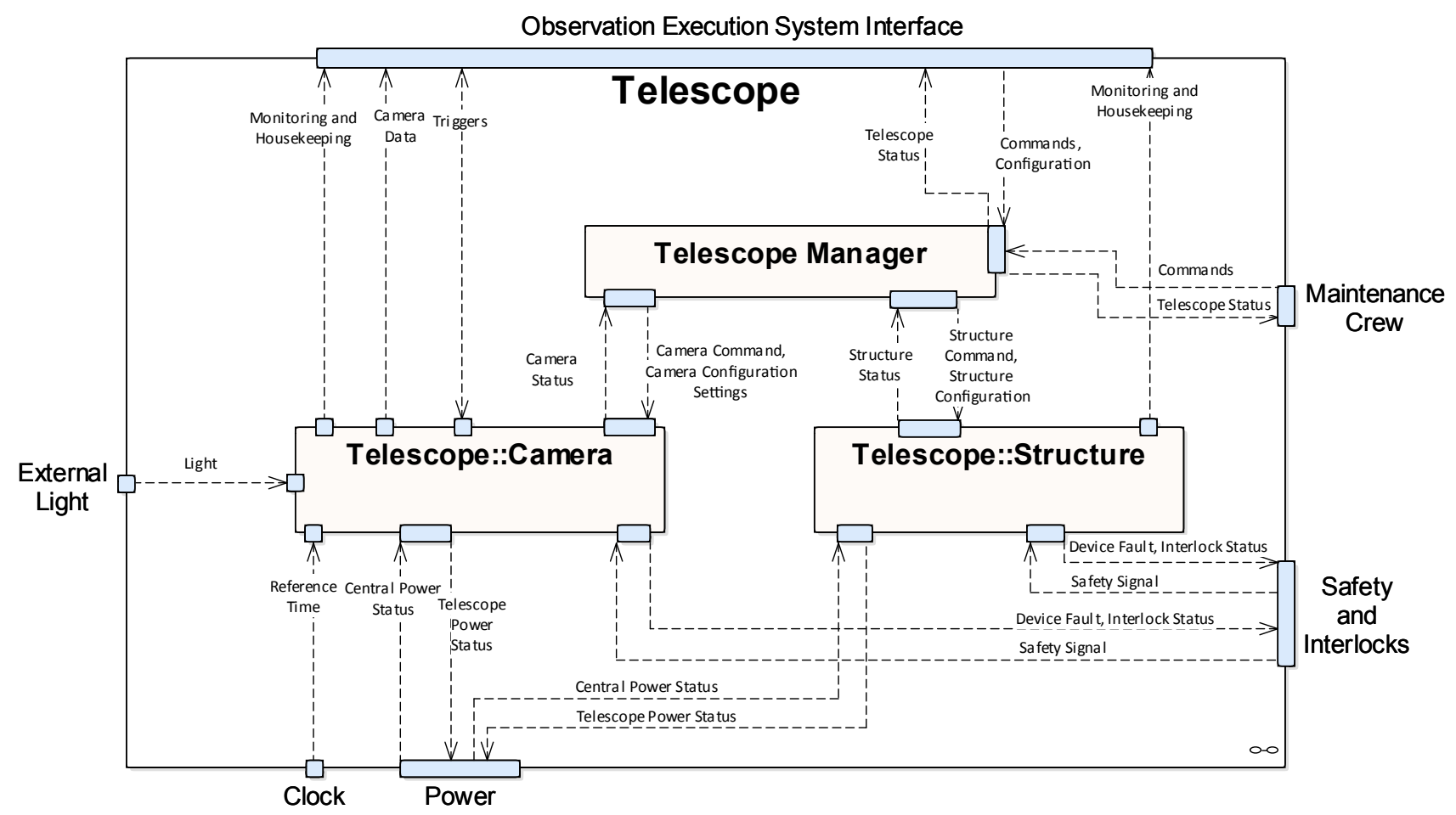

Figure 1. Simplified view of the main component of a CTA telescope, as well as the main connection to surrounding systems and environment. The blue rectangles are ports and represent connection to other systems and users. The dashed arrows represent flow of data or stimuli from or to the telescope elements.

4. Select those use cases that require further specification. In general, specialized use cases do not require further details beyond the short description and the "generalization" relationship with a fully detailed general use case. Also, in some use cases, we felt we could not give more details at the time of writing the use cases due to missing input ${ }^{\ddagger}$ (e.g. specific maintenance prescriptions). For each of the selected use cases, we:

(a) Fully furnish the use case according to the categories in Table 1. This means to identify the pre- and post-conditions and the individual steps in the use cases scenarios.

(b) Assign a tentative state for the telescope (see later).

5. Iterate a new version of the UC from the start.

After each iteration, a new version of the use case document was generated using the EA document generation facilities. Telescope experts were consulted in face-to-face meetings and via remote communication through the iterations. The states associated to each use case step was confronted with the allowed list of telescope states, and adaptations were done until both converged.

The telescope is considered as a grey box in our analysis, i.e., some UCs consider the telescope behaviour as a whole, whereas for other use cases we specify the behaviour of the structure and the camera. This dual level of granularity was necessary both to establish efficient communication with hardware experts (usually different personnel work in Camera and in Structure groups), and in order to determine the role of the Telescope Manager component (see Section 2).

\footnotetext{
${ }^{\ddagger}$ These use cases will be furnished in future updates, when the missing input becomes available.
} 
Table 1. Use Case Template.

\begin{tabular}{|l|l|}
\hline Pre-conditions & Conditions that both the system and actors must have in the moment the UC starts. \\
\hline Trigger & Event that causes the UC to start. \\
\hline Frequency & An indication of how often the UCs happens. \\
\hline Goal & Goal of the UC. \\
\hline Actors and Systems & Actors and systems interacting in the UC. \\
\hline Main Scenario & Default sequence of events (the "happy path"). \\
\hline Alternative path & $\begin{array}{l}\text { An alternative event path branching at a step of the main scenario due to expected } \\
\text { conditions. This path may join back to the main scenario at a given step, or at the end. }\end{array}$ \\
\hline Exception path & $\begin{array}{l}\text { An alternative event path branching at a step of the main scenario due to a problem } \\
\text { or unexpected event. This path may join back to the main scenario at a given step, } \\
\text { or at the end. }\end{array}$ \\
\hline Success guarantee & State and conditions of the system when the main scenario happens without problem. \\
\hline Minimal guarantee & $\begin{array}{l}\text { Absolute minimal conditions that the system must be in after the UC, } \\
\text { no matter what failed. }\end{array}$ \\
\hline
\end{tabular}

\section{STATUS}

The total number of CTA telescope use cases amounts to 72, about half of which are fully furnished. Table 2 shows, as an example, one of the UCs we have produced. The UCs have been classified according to the following categories:

- Start up and Shut down

- Control and Acquisition

- Performance and Monitoring

- Calibration

- Safety and Alarms

- Engineering

The complexity of the created UCs ranges from some cases which require more than a whole page of text, describing ten scenario steps and more than eight alternative/exception paths, to those in which just a couple of lines of text is sufficient to describe it.

The UCs served us to identify interfaces and interactions of the telescopes with other systems. Many of the interactions of the telescope happen with the OES system, but we found also interactions with other systems such as the SAS. Additionally, we identified several direct operations to be carried out by observatory personnel such as the Operator and Maintenance Technician, associating these operations to the personnel roles.

Additional use cases required for the telescope assembly, integration and verification (AIV) process are specific to each telescope model and to be created by the telescope development teams.

\section{LESSONS LEARNED}

The work presented here has been an important facilitator in the communications between the CTAO architecture team and the groups developing the CTA telescopes. The UCs have been used as a tool to refine the telescope and OES requirements; they have served to elicit missing functional requirements, and to explain further already 
Table 2. Example of a CTA Telescope Use Case.

\section{Perform Telescope Pointing Calibration}

Summary and Scope: OES instructs Telescope to get ready for pointing calibration. OES creates a list of pointing positions (typically stars) that the Instrument Scientist can revise or modify. OES provides sequentially new positions and issues pointing measurement acquisition for all the position in the list. Instrument Scientist reviews the results and eventually creates a new pointing model, uploading it to the OSS configuration database.

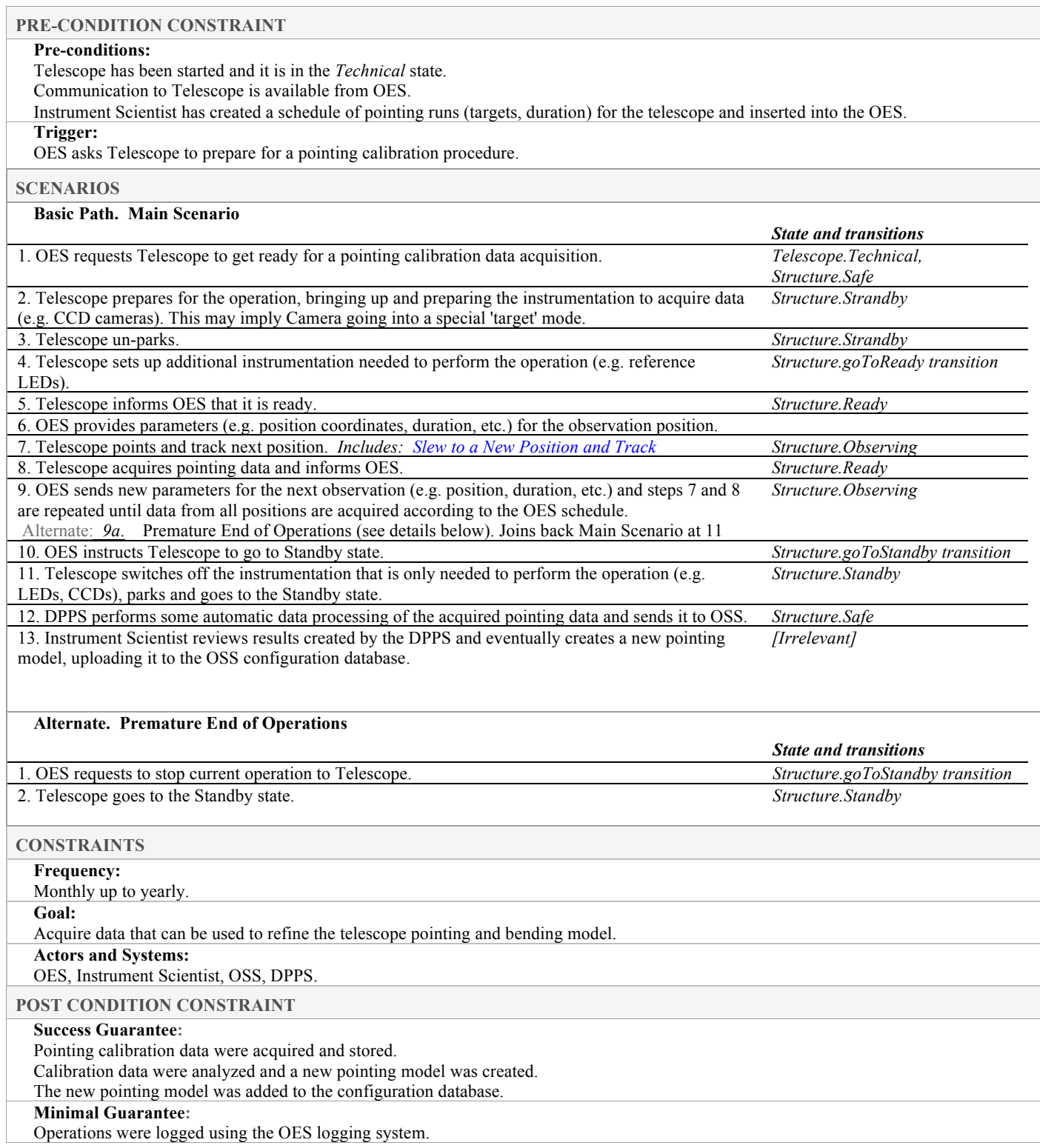

Notes: This use case describes how a CTA telescope is used by the OES to acquire data that is later used by an Instrument Scientist, using the DPPS, to improve the pointing of this particular telescope. The use case has a main scenario and a single alternative path, Premature End of Operations. This use case "includes" another telescope use case, Slew to a New Position and Track. The state of the telescope as a whole or of the telescope structure is indicated in the right-hand column. 


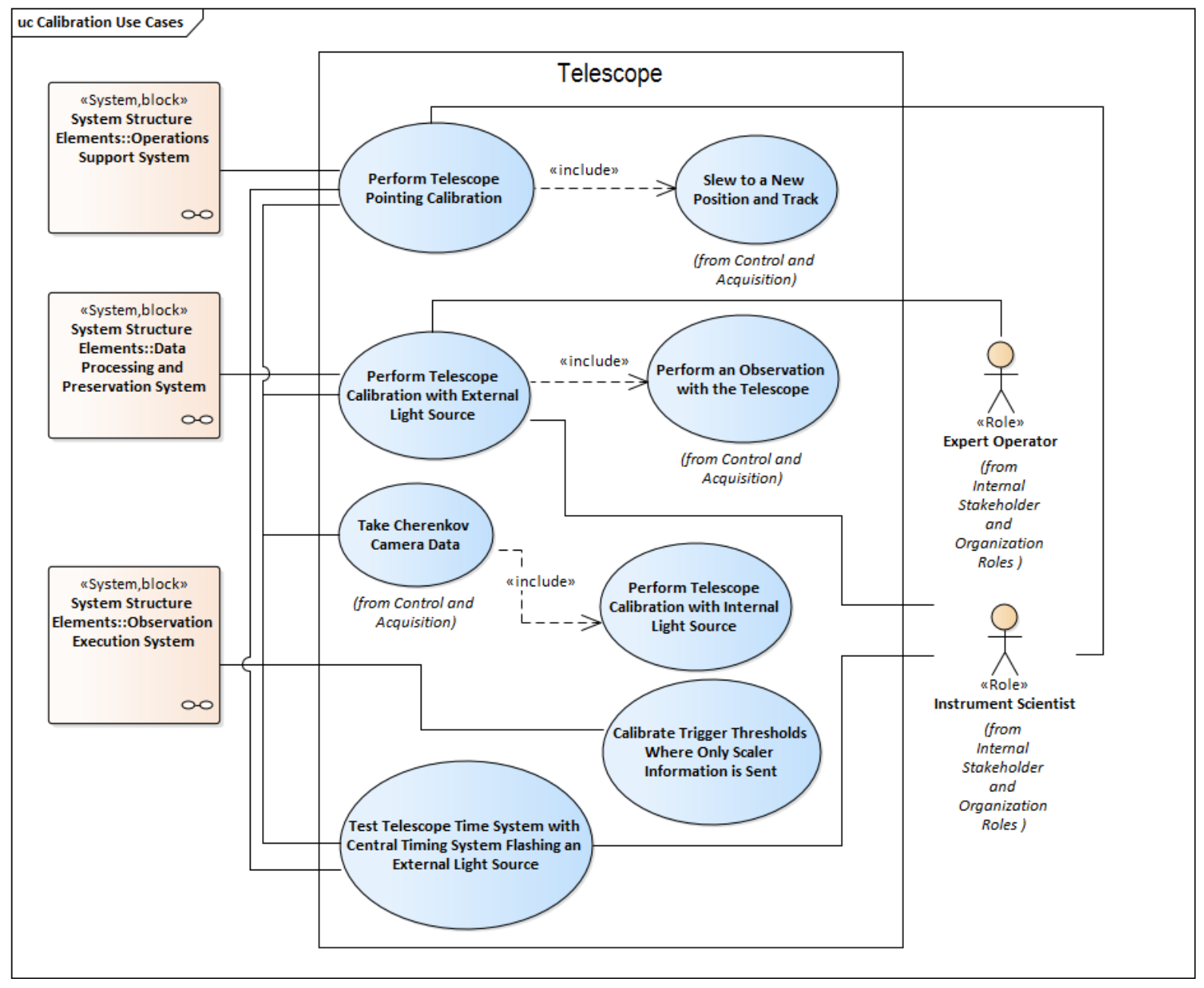

Figure 2. Example of one of the UML use case diagrams used for the analysis of the telescope use cases. In this particular example, we show the UCs associated to calibration operations. If we pick the example of the Perform Telescope Pointing Calibration, this diagram shows that three systems and one human actor are playing a role in the scenario and that the UC uses another one, Slew a new Position and Track.

existing ones. These UCs also provide a solid basis to create a generic software interface from the OES to the CTA telescopes.

Our conclusion is that such systematic methodologies enhance the communication between teams and streamline the convergence to a common vision of the system between the system architects and managers and the software and hardware providers. In the course of our discussions with the instruments experts, we sometimes discovered that certain incompatible assumptions had been taken by the teams designing the different aspects of the system. To illustrate this with an example, the assumption of the system behaviour when emergency stops close to the telescope are activated were different among the hardware and central software teams. After our discussions, a homogeneous approach has been specified, and a common view exists now in the project.

The content of the UCs presented in this paper is fully integrated in a EA modelling file, which is stored in a cloud database. Thanks to this cloud facility, several designers could work in parallel in the modelling of the CTAO architecture and in the creation of these use cases, providing traces with associated architecture elements. EA provides convenient ways to publish the contents of the use cases as Microsoft Word and PDF format using the EA document generation facilities. 


\section{OUTLOOK}

The current set of CTA generic telescope UCs is still under review within the CTAO, but close to being finalized. We may still need to add some additional use cases, as well as to incorporate a few more details to the existing ones. Individual telescope AIV UCs, which are specific for each type, must be be harmonized with the generic ones. In addition, the technical interface of OES and the Telescopes, which is currently under construction, will be tested and validated based on this work.

\section{ACKNOWLEDGMENTS}

The authors acknowledge the support of both CTAO gGmbH personnel and CTA Telescope Structure and Camera team members. We acknowledge the useful comments of CTA consortium, which was instrumental for the definition of the use cases. We gratefully acknowledge financial support from the agencies and organizations listed here: http://www.cta-observatory.org/consortium

\section{REFERENCES}

[1] Acharya, B., Actis, M., Aghajani, T., et al., "Introducing the CTA concept," Astroparticle Physics 43, 3 (2014).

[2] Aharonian, F., Akhperjanian, A., Bazer-Bachi, A., et al., "Observations of the Crab nebula with HESS," $A \& A$ 457, 899 (2006).

[3] Albert, J., Aliu, E., Anderhub, H., et al., "VHE Gamma-Ray Observation of the Crab Nebula and its Pulsar with the magic," ApJ 674, 1037 (2008).

[4] Acciari, V. A., Beilicke, M., Blaylock, G., et al., "VERITAS Observations of the Gamma-Ray Binary LS I +61 303," ApJ 679, 1427 (2008).

[5] Fuessling, M. et al., "A system architecture approach for the Cherenkov Telescope Array," in [These proceedings], (2018).

[6] Oya, I., F, M., Antonino, P., et al., "Designing and prototyping the control system for the cherenkov telescope array," in [ACAT 2017 Procs.], Contribution 42 (2017).

[7] Actis, M., Agnetta, G., Aharonian, F., et al., "Design Concepts for the Cherenkov Telescope Array CTA, An Advanced Facility for Ground-Based High-Energy Gamma-Ray Astronomy," Experimental Astronomy 32, 193 (2011).

[8] Hillas, M., "Cerenkov light images of EAS produced by primary gamma rays and by nuclei," in [Proc. 19th ICRC, La Jolla], 3, 445 (1985).

[9] Cockburn, A., [Writing Effective Use Cases], Addison-Wesley Professional, Boston (2000 (first edition)). 